\title{
Uni-polar or bi-polar scales of judgment
}

\author{
M. E. TRESSELT
}

NEW YORK UNIVERSITY

\section{Abstract}

A study was made to try to determine whether Ss proceeded from rankings of 1-15 in an orderly fashion when asked to make preferential judgments of colors and types of music or whether there was a characteristic pattern to these rankings of two types of stimuli. Forty Ss were asked to rank from 1-15 an arbitrary listing of 15 colors and of 15 types of music and also to indicate the order of the selections; i.e., was the carmine red judged as second most preferred but only after 5 other judgments had been made? The results show the same type of curve for both materials; both suggesting a discontinuity in the activity of judgment.

\section{Problem}

The concept of a continuum on which scale points go from one extreme through a neutral or indifferent zone and then to the other extreme has been generally accepted, especially by the psychophysicists and those interested in judgmental scales. The basis for this assumption is a realistic one if one considers that in most instances, a $\mathrm{S}$ may utilize end anchoring in his judgments, i.e., given a sheet of paper $8-1 / 2 \times 11$ in, the 10 in line will be called a "long" line and the $1 / 2$ in line, a "short" line with a 5 in line perhaps being called "medium." In most experiments utilizing linear measures, not all stimuli are given at once but the method of comparison or the method of absolute judgments are utilized. By using these techniques, it is impossible to know whether a $\mathrm{S}$ is actually using a full continuum; a half-continuum; or perhaps two separate continua. In order to study this problem, it was decided to utilize a relatively easy situation of preferences.

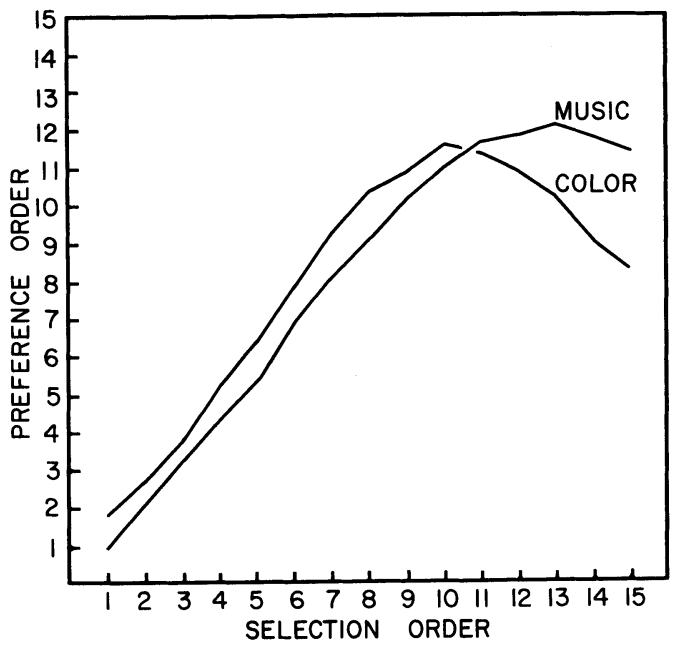

Fig. 1. The absolute selection choice of color and musical type (selection order) plotted against the ordinal position in time of the preference (preference order).

\section{Procedare}

A total of $40 \mathrm{Ss}$ was given mimeographed sheets of two different subject matters. In one instance, the $\mathrm{S}$ was given a sheet with 15 arbitrary colors (carmine red, royal blue, Irish green, canary yellow, purple, pink, baby blue, light green, lemon yellow, violet, flamingo or red orange, turquoise or blue-green, chartreuse or yellow green, brown and fuchsia or red violet) and asked to select the one they liked best and write it next to number 1 on the sheet, the one they liked next best to number 2, etc. As they selected the colors of their choices, they were asked to indicate whether it was the first one placed, the second, etc. by indicating the appropriate number after the color name. A second sheet had the same instructions but concerned itself with 15 types of music ('bop' jazz, cantatas, chamber music, concertos, Dixieland jazz, folk, Gregorian chants, hymns, lieder, operas, piano sonatas, popular, symphonies, violin sonatas, waltzes).

\section{Results}

Figure 1 shows the absolute selection choice of color (selection order) plotted against the ordinal position in time of the preferences (preference order), i.e., the preferred color, number one, was usually written down first by the majority of Ss. To be exact, the ordinal position in time of the first choice color was 1.80. There were some Ss obviously who ranked the least preferred colors first. The 15th color, or least preferred color was generally the 8 th or 9 th selection made. The same kind of curve seems to be indicated by the results in music.

Theoretically, then Ss performed these tasks quite differently but most of them on the average seem to be using two different scales, one extending from like to neutral and the other from dislike to neutral. No one started from the neutral position and worked towards the extremes, so it seems reasonable to conclude that end-anchoring was operating but for two different continua.

\section{Diseussion}

The possibility of two different criteria being present in some experimental situations and not in others may account for some of the discrepant findings in judgmental research. In research where all variations of the stimuli are known, assimilation as well as contrast may be effected depending on which type of positive-to-neutral or negative-to-neutral continuum is being utilized. It would seem a necessary follow-up to investigate individual performances and scales to better understand deviations from phenomena as Helson's Adaptation Theory or Sherif and Hovland's concept of assimilation and contrast or even cognitive dissonance. 
Reply to Lockhead by C. W. Eriksen and J. S. Lappin

In the interests of logical precision we would point out that the demonstration of an alternative interpretation or model for a set of data does not in itself prove other models or interpretations to be "incorrect." Choices between models are based upon other considerations such as their simplicity, ability to integrate other phenomena, and/or their yielding different predictions that can be tested empirically. It is unfortunate that Dr. Lockhead did not replicate our experiment completely or at least replicate the bare minimum of conditions that are logically necessary to compare our model and the one he proposes.

To adequately compare the two models Lockhead should have included the additional condition where there were six simultaneous occurrences of the same form in a presentation. This condition and his condition of no redundancy but where $\mathrm{S}$ knew the form would appear at the $100^{\prime}$ clock position both have position certainty. His model would predict the same identification accuracy for these two conditions whereas our model would predict a higher probability of identification for the condition with the redundancy. Unfortunately he chose to replicate only the condition where both models make essentially the same prediction.

Since Lockhead does not provide confidence rating data, we cannot apply exactly our probability model to his results. However an approximation can be applied that will at most overestimate by only a couple of percentage points the hit rate (HR) for conditions containing the redundant form occurrences. Using Lockhead's data for HR with only one occurrence of the form and position uncertainty, we would break this HR (42\%) into two components-state A where $\mathrm{S}$ perceived the form and state B where he guessed correctly. With a HR of $42 \% \mathrm{~S}$ must have guessed wrong $58 \%$ of the time. With a three alternative forced-choice situation the best estimate of his correct guesses is $29 \%$ leaving 13 as the percentage of the trials he actually perceived the stimulus. The probability that $\mathrm{S}$ would fail to perceive on two independent chances (two simultaneous occurrences of the form) is $\mathrm{P}^{2} \mathrm{~B}$ or $.87^{2}=.76$. The probability that he perceived one or both of the forms is $1-\mathrm{P}^{2} \mathrm{~B}$ or .24 . Since he would guess with an a priori probability of being correct $1 / 3$ of the $76 \%$ of the trials he failed to perceive, the value of $25 \%$ must be added to the $24 \%$ to obtain his predicted HR of $49 \%$.

With six independent chances the probability $S$ fails to perceive on any of them is $\mathrm{P}^{6} \mathrm{~B}$ or $.87^{6}=.43$. The probability he perceives one or more of the six occurrences is $1-\mathrm{P}^{6} \mathrm{~B}$ or $57 \%$. When the number of correct guesses is added to this value of $57 \%$, i.e., $1 / 3$ of $47 \%$, the predicted HR is found to be $73 \%$.

As is seen, our predicted HR for the two form occurrence $(49 \%)$ is very close to that obtained by Lockhead (48\%). Unfortunately his prediction for this case is essentially the same (50\%) and thus his experimentdoes not provide a test of the two different interpretations of the underlying process. Had he included the six occurrence condition, a clear test would obtain since he would still predict a HR of $50 \%$ whereas our model predicts $73 \%$.

Lockhead has also overlooked a very important aspect of our data. The function relating HR to number of same forms presented in a single presentation has marked negative acceleration. The average increase in HR as the number of occurrences of the form is increased from one to two, is slightly over $10 \%$, but the HR increase as the number of occurrences is increased from four to six, is less than $2 \%$ (the difference between these differences is significant at $p<.01$ ). Lockhead's explanation of our data would require a linear function. From his data the HR is $50 \%$ when attention is an the position where the form appears. When this HR is corrected for guessing, the percent of time sperceives the form with "attention" properly directed, is $25 \%$. Thus with six possible positions $\mathrm{S}$ would be expected to perceive the form $1 / 6$ of $25 \%$ of the time $(4.2 \%)$ and guess on the remaining $95.8 \%$ of the trials. With credit for $1 / 3$ correct guesses his HR for one form occurrence in six possible positions would be $36.1 \%$. Similar computations for 2, 3, 4, and 5 simultaneous form occurrences show that Lockhead's model predicts corresponding HRs of $38.9,41.7,44.4$ and 47.2 percent. As is seen, this model describes a linear function for $\mathrm{HR}$ and number of form occurrences.

One final point concerns why Lockhead obtains a higher HR with only one form occurrence when S can "attend" the position where the form occurs. We would suggest that Lockhead's "attention" is nothing more than visual fixation since his procedure provides no control for where the eyes are fixated when the tachistoscope flashes. As is generally known the fovea varies markedly in acuity even within 1 to $2^{\circ}$ of its center. We have recorded eye movements and changes in fixation during tachistoscopic presentations. It can be readily verified that even highly practiced Ss cannot resist change in their fixation from the designated fixation point to the expected position of the stimulus. If the $\mathrm{S}$ initiates the tachistoscope presentati on, apparently his internal decision to trigger the switch is a signal for changing fixation to the expected stimulus location. The latency of the eye movement seems to be shorter than the finger activating the switch with the result that the eyes have moved to the expected stimulus position before the tachistoscope flashes. If the $\mathrm{E}$ initiates the tachistoscope, his ready signal serves to cue the S's change in fixation. Only with stimulus position uncertainty of three or more positions can Ss generally be relied upon to maintain fixation on the designated fixation point until the stimulus is presented.

If Lockhead's Ss were shifting their fixation point slightly from the designated one to the position where the stimulus was anticipated, the increased visual acuity for the central foveal fixation might be expected to result in an $8 \%$ increase in form identification.

For article by G. R. Lockhead see page 319 . 